\title{
Dynamical arrest: interplay of the glass and of the gel transitions
}

\author{
Nagi Khali1, ${ }^{a \ddagger}$, Antonio Coniglio $^{b}$, Antonio de Candia ${ }^{b, d}$, Annalisa Fierro ${ }^{b}$, and Massimo Pica Ciamarra ${ }^{b, c}$
}

\section{Received Xth XXXXXXXXXX 20XX, Accepted Xth XXXXXXXXX 20XX}

First published on the web Xth XXXXXXXXXX 200X

\section{DOI: $10.1039 / b 000000 x$}

'The structural arrest of a polymeric suspension might be driven by an increase of the cross-linker concentration, that drives the gel transition, as well as by an increase of the polymer density, that induces a glass transition. These dynamical continuous '(gel) and discontinuous (glass) transitions might interfere, since the glass transition might occur within the gel phase, and the 'gel transition might be induced in a polymer suspension with glassy features. Here we study the interplay of these transitions by investigating via event-driven molecular dynamics simulation the relaxation dynamics of a polymeric suspension as a function 'of the cross-linker concentration and the monomer volume fraction. We show that the slow dynamics within the gel phase is 'characterized by a long sub-diffusive regime, which is due both to the crowding as well as to the presence of a percolating cluster. In this regime, the transition of structural arrest is found to occur either along the gel or along the glass line, depending on the length scale at which the dynamics is probed. Where the two line meet there is no apparent sign of higher order dynamical singularity. Logarithmic behavior typical of $A_{3}$ singularity appear inside the gel phase along the glass transition line. These 'findings seem to be related to the results of the mode coupling theory for the $F_{13}$ schematic model.

\section{Introduction}

'A polymeric suspension might behave as a hard sphere system, when no cross-linkers are added to it, as its dynamics 'slows down as the concentration increases. In this case, a transition of structural arrest, the glass transition, occurs when the volume fraction $\phi$ overcomes a critical threshold, $\phi_{\text {glass }}$. This transition marks the arrest of the dynamics at all relevant length scales, for wavevectors ranging from $k_{\min }=2 \pi / L$ to $k_{\max }=2 \pi / \sigma$, with $L$ and $\sigma$ sizes of the system and of the particles. The glass transition is a discontinuous dynamical phase transition because its order parameter, known as nonergodicity parameter and defined as the infinite time limit of relaxation functions, is zero below the transition but acquires a finite value at the transition. Polymeric suspensions may also undergo a different kind of structural arrest transition, the gel transition. This occurs at fixed volume fraction on in'creasing the cross-linker concentration, which is related to the probability that two close monomers are permanently bonded. This concentration can be tuned, for instance, by radiation as

${ }^{a}$ Departamento de Física, Universidad de Extremadura, E-06071 Badajoz, Spain

${ }^{b}$ CNR-SPIN, Dipartimento di Scienze Fisiche, University of Napoli Federico II, Italy

${ }^{c}$ Division of Physics and Applied Physics, School of Physical and Mathematical Sciences, Nanyang Technological University, Singapore

${ }^{d}$ INFN, Sezione di Napoli, Complesso Universitario di Monte S. Angelo, Via Cintia, Edificio 6, 80126 Naples, Italy in light induced polymerization processes (e.g., dental filling pastes) or by heat (e.g., cooking). On increasing the bonding probability, one drives the system across a gel transition, where a spanning cluster of connected monomers emerges. This leads to a gel transition line $\phi_{\text {gel }}(p)$ in the $p-\phi$ plane. This structural transition also corresponds to a dynamical arrest transition, as the dynamics becomes frozen on the smallest wavevector $k_{\min }$ being the spanning cluster unable to diffuse. Contrary to the glass transition, this is a continuous transition, as the order parameter continuously increases as one enters the gel phase on increasing $p$ or $\phi$.

The interference of different transitions of dynamical arrest have been previously investigated, via model coupling theory, in systems of particles interacting with a hard core repulsion competing with a very short range attraction. These systems show an attractive and a repulsive glass transition, both of them discontinuous, and mode coupling theory predicts that their interference leads to an $A_{3}$ high order singularity characterized by logarithmic decay of the correlation functions $1-3$. These predictions were verified in different colloidal systems ${ }^{4-9}$, both experimentally and numerically. Similar findings were also observed in Ref. ${ }^{10}$, where the dynamics of a physical gel was numerically studied at high densities where gelation and glass transition interfere.

In this paper we study the interference of a continuous and of a discontinuous transition of structural arrest focusing on a system with permanent bonds where, as illustrated in Fig. 1.

This journal is @ The Royal Society of Chemistry [year] Journal Name, 2010, [vol], 1-7 | 1 


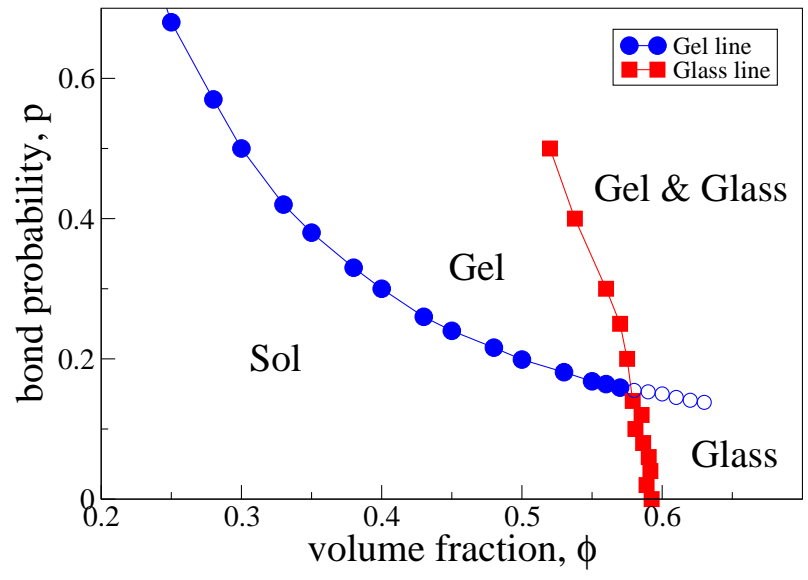

Fig. 1 Structural arrest diagram as a function of the volume fraction, $\phi$, and of the bonding probability, $p$, illustrating the interplay of the gel and the glass transition lines. The gel line is determined via percolative analysis, after introducing bonds with probability $p$ in an equilibrium (full symbols) or in an out-of-equilibrium (open symbols) monomer suspension. The glass line is defined as that where the extrapolated diffusion coefficient vanishes. Only points up to $p=0.5 \phi=0.54$ are shown, where the self Intermediate Scattering Function (sISF) exhibits a logarithmic behavior, which we associate to an $A_{3}$ singularity as predicted by the $F_{13}$ mode coupling schematic model (see text and Fig. 8). Solid lines are guides to the eye.

the gel $\phi_{\mathrm{gel}}(p)$ and the glass transition line $\phi_{\mathrm{glass}}(p)$ intersect. We address some questions raised by the presence of these two transition lines. First, we note that the gel transition corresponds to structural arrest occurring at the largest $\left(k_{\min }\right)$ scale, while all scales, including the smallest one $\left(k_{\max }\right)$, are relevant in the structural arrest corresponding to the glass transition. Therefore, we consider the length scale dependence of the transition of structural arrest. Second, we note that the glass transition line enters in the gel phase at a point where both continuous and discontinuous transition occur. Hence we expect that the model system here studied might have some analogies with the mode coupling theory for schematic $F_{13}$ model $^{2}$, which gives two arrested lines: a continuous transition, which meets a discontinuous one. This discontinuous transition ends on a high order critical point $\left(A_{3}\right.$ singularity) characterized by logarithm decay $\underline{\underline{1}-\frac{3}{n}}$ of the relaxation functions. Topologically the phase diagram is similar to the one found here (Fig. 1). Consistently with mode coupling theory, we find logarithmic behavior at a point well in the gel phase along the glass line.

\section{Numerical model}

We perform event driven molecular dynamics simulations 11,12 of a 50:50 binary mixture of $N=10^{3}$ hard spheres
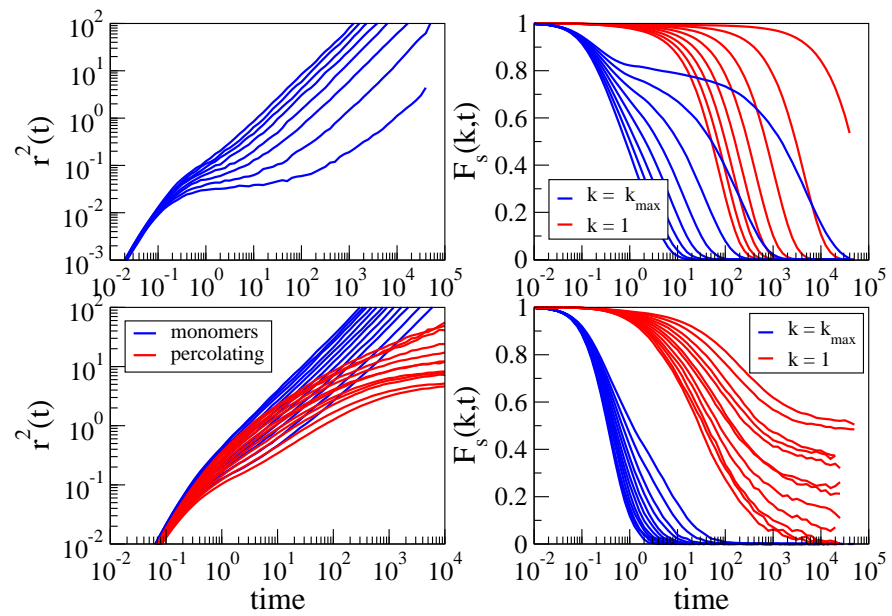

Fig. 2 Mean square displacement (left) and sISF (right). Top panel: on approaching the glass transition at $p=0$, with volume fraction from $\phi=0.52$ to $\phi=0.59$ (from left to right). Bottom panel: on entering the gel phase at $p=0.3\left(\phi_{\text {gel }} \approx 0.4\right)$, with volume fraction from $\phi=0.4$ to $\phi=0.51$ (from left to right).

(monomers) of mass $m$ and diameters $\sigma$ and $1.4 \sigma$, in a box of size $L$ with periodic boundary conditions. The chosen size ratio is known to effectively prevent crystallization $13-16$. The volume fraction $\phi=N v / L^{3}$, where $v$ is the average particle volume, is tuned by changing the size $L$ of the box. The mass $m$, the diameter $\sigma$ of the smaller particles, and the temperature $T$, fix our mass, length and energy scales, while the time unit is $\sqrt{m \sigma^{2} / T}$. After thermal equilibration at the desired volume fraction, permanent bonds are introduced with probability $p$ between any pair of particles separated by less than $1.5 \sigma$. A bond corresponds to an infinite square well potential, extending from $\sigma$ to $1.5 \sigma$. The procedure we use to insert the bonds mimics a light-induced polymerization process, as the number of bonds depends on both $p$ and $\phi$.

Using the percolation approach, we identify the gel phase as the state in which a percolating cluster is present $\stackrel{17,18}{ }$, and the gelation transition as the percolation line. A standard finitesize scaling analysis of the mean cluster size $\frac{19}{}$ is applied to identify the percolation line. The resulting line is illustrated in Fig. 1. The gel transition occurs at a critical bond probability $p_{\text {gel }}(\phi)$ decreasing with $\phi$.

\section{Standard glass and gel behaviours}

Since we are interested in the interplay of the gel and of the glass transition lines, we start by shortly reviewing the main features of the slowing down of the dynamics when these transitions do not interfere. First, we consider the transition at zero bonding probability, where our system reduces to a hard sphere suspension and undergoes a glass transition as the 
volume fraction increases. This crowding induced dynamical transition is characterized by well known signatures in the mean square displacement, $r^{2}(t)$, and in the self intermediate scattering function (sISF), $F_{S}(k, t)$, defined respectively by:

$$
\left\langle r^{2}(t)\right\rangle=\frac{2}{N} \sum_{i=1}^{N / 2} r_{i}^{2}(t)
$$

and

$$
F_{S}(k, t)=\frac{2}{N} \sum_{i=1}^{N / 2} e^{i k\left(r_{i}(t)-r_{i}(0)\right)},
$$

where the sums extend only to the larger particles. These are reviewed in the top row of Fig. 2. At high densities, particles rattle in the cages formed by their neighbours before entering the diffusive regime. Accordingly, the mean square displacement develops a plateau at intermediate times, known as Debye-Weller factor. This plateau becomes longer and longer as the density increases. The diffusivity $D=\lim _{t \rightarrow \infty} r^{2}(t) / 6 t$ decreases on increasing $\phi$, and vanishes at the glass transition. Consistently with the behaviour of $r^{2}(t)$, the relaxation function at large $k$ develops a two step decay, the first one ( $\beta$ relaxation) associated to the rattling within the cages, the second one $(\alpha)$ to the onset of diffusive motion. The $\beta$ relaxation becomes less and less visible as $k$ decreases, and length scales larger than those associated to the typical vibrational motion are probed. At the glass transition, only the $\beta$ relaxation occurs, and $F_{s}(k, t)$ asymptotically reaches a finite value, known as non-ergodicity parameter $f_{k}$. The glass transition is a discontinuous phase transition as $f_{k}$ varies discontinuously across the transition.

We now consider the transition at high $p$, where on increasing $\phi$ the system undergoes a gel transition at a volume fraction $\phi_{\text {gel }} \ll \phi_{\text {glass }}$, so that the two transitions do not interfere. The typical behaviour of $r^{2}(t)$ and of $F_{s}(k, t)$ on approaching this transition is illustrated in Fig. 2 (bottom row). Due to the presence of bonds, in the system there are clusters with a different size $s$. In the long time limit, each cluster diffuses with a diffusivity decreasing with $s$. Accordingly, at long times the mean square displacement is essentially fixed by that of the fastest clusters, the monomers. Since the volume fraction is small, the diffusivity of the monomers is only slightly affected by the volume fraction and has not special features at the gel transition, above which the monomers diffuse within the percolating polymer network. On the contrary the largest cluster, whose mean square displacement is also illustrated in Fig. 2, slows down on approaching the gel transition, and indeed its diffusivity vanishes at the transition (where the largest cluster percolates). We note that, on increasing $\phi$, the mean square displacement of the largest cluster does not reach the diffusive regime on the time scale of our simulations. Indeed, it has three contributions: the first is due to the vibrations of the particles in the cages formed by nearest neighbors, the second to the fluctuations of the cluster structure, and the third to the diffusion of its center of mass. Of these, only the third reaches the diffusive regime at long times. At the gel transition, when the diffusion of the center of mass of the largest cluster vanishes, the mean square displacement remains subdiffusive at all times, while inside the gel it eventually reaches a plateau.

The behaviour of the sISF critically depends on the length scale at which it is probed. At small scales, that is for $k=k_{\max }$, it does not exhibit any particular feature on approaching the gel transition. On the other hand, at larger length scales, $k=k_{\min }$, the relaxation time diverges when $\phi$ approaches $\phi_{\text {gel }}(p)$. At the transition, the correlator decays in time as a power law, while inside the gel asymptotically tends to a plateau, $f_{k}$, the non-ergodicity parameter, that is zero at the the transition, and continuously grows on entering the gel phase. The growth is continuous as the value of $f_{k}$ is related to the density of the particles in the percolating cluster, and of the monomers that are trapped in the percolating cluster. The gel transition therefore induces a continuous dynamical phase transition. These properties are found in gelling systems both experimentally $20-22$ and numerically $\underline{2}-26$. In Ref. ${ }^{27,28}$, deep analogies of gelation and spin glass transition was suggested.

\section{Dynamics in the gel phase}

We now consider how the features of the relaxation process changes as the volume fraction increases, and the system enters the gel phase and approaches the glass transition. This is identified as the volume fraction where the extrapolated diffusivity of the monomers vanishes (see Fig. 11). In this section we focus on a high enough value of the bond probability, $p=0.3$, for which $\phi_{\text {gel }} \approx 0.4$ is sensible smaller than $\phi_{\text {glass }} \approx 0.58$.

\subsection{Mean square displacement}

In the gel phase, the mean square displacement at intermediate times shows a subdiffusive regime, similar to that observed in the slow dynamics of colloidal particles diffusing in a matrix of disordered hard sphere obstacles $\underline{30}$. We have characterized it by investigating the time dependence of the subdiffusive exponent $q$, defined by $q=d \log r^{2}(t) / d \log t$. Data are illustrated in Fig. 3 This exponent exhibits a crossover from the ballistic short time value $q=2$, to the diffusive long time value, $q=1$. At intermediate times, a subdiffusive behaviour is found, $q<1$, which becomes prominent as the volume fraction increases. Further increasing the volume fraction, very peculiar features appear, as the exponent $q$ might also exhibit two minima. This behaviour is rationalized considering that the mean square displacement, Eq. (1), has contributions from particles belonging to different finite clusters of size $s$, as well as from the spanning cluster $(s=\infty$, in the thermodynamic

This journal is @ The Royal Society of Chemistry [year]

Journal Name, 2010, [vol], 1-7 | 3 


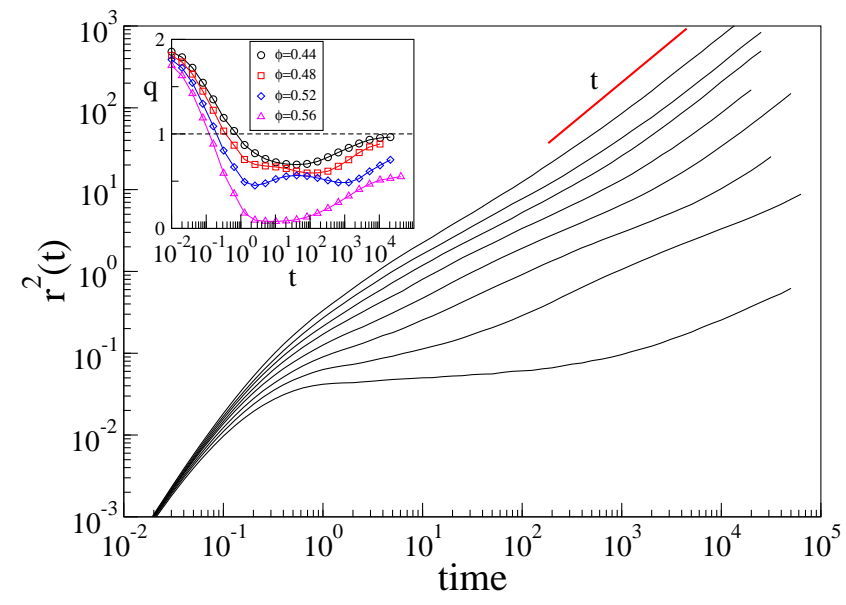

Fig. 3 Mean square displacement in the gel phase, for $p=0.3$ and $\phi$ from 0.42 to 0.56 (from top to bottom). In the inset, the subdiffusive exponent $q=d \log r^{2}(t) / d \log t$, as function of time for $p=0.3$ and $\phi=0.44,0.48,0.52,0.56$.

limit), as

$$
\left\langle r^{2}(t)\right\rangle=\frac{1}{N} \sum_{s} s n_{s}\left\langle r_{s}^{2}(t)\right\rangle
$$

where the sum runs over the cluster size $s,\left\langle r_{s}^{2}(t)\right\rangle$ is the mean square displacement of clusters of size $s$, and $n_{s}$ is the number of cluster with size $s$. The cluster size distribution $n_{s}$ and the mean square displacement for different $s$ are illustrated in Fig. [4 for $p=0.3$ and $\phi=0.54$. The figure clarifies that two phenomena lead to the subdiffusive behaviour. The first one is the crowding induced cage effect typical of glasses, which involves both particles belonging to finite clusters, as well as particles belonging to the percolating cluster. The second one only involves the particles of the percolating cluster, whose mean square displacement reaches a plateau at long times, as the percolating cluster is unable to diffuse. The coexistence of these two effects, whose relevant importance is fixed by $\phi$ and $p$, leads to the unusual features of the mean square displacement plotted in Fig. 3 .

As we have observed above, the diffusive problem in the gel phase shares some similarities with the much investigated problem of single-particle diffusion in a matrix of disordered hard sphere obstacles ${ }^{29} \underline{-31}$, where single particles are considered to move in a frozen environment. Conversely, in the present case, monomers and finite clusters diffuse in a fluctuating environment, as the percolating clusters may vibrate. These fluctuations affect the diffusion of free particles as we see in Fig. 5. where the mean square displacement obtained in the actual gel is compared with that obtained by freezing the percolating cluster. The figure clarifies that the fluctuations of the percolating cluster strongly influence the diffusivity of the particles. For instance, at $p=0.25, \phi=0.55$, the system is

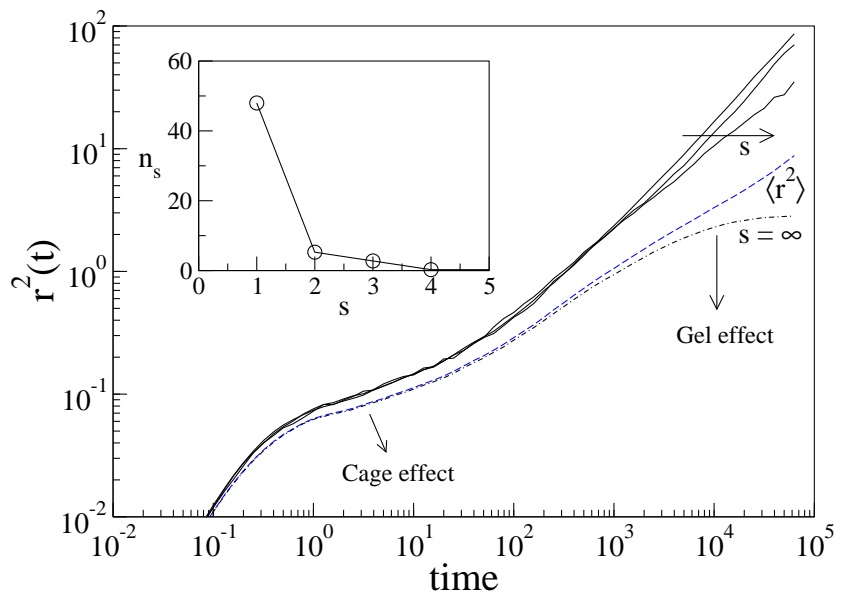

Fig. 4 Main panel: mean square displacement averaged over all the particles, $\left\langle r^{2}\right\rangle$, over particles belonging to finite clusters of size $s=1,2,3$, and over particles belonging to the percolating clusters, for $p=0.3$ and $\phi=0.54$. Inset: size distribution of finite clusters for the same values of $p$ and $\phi$. There is also a percolating cluster, with $s \approx 930$ particles.

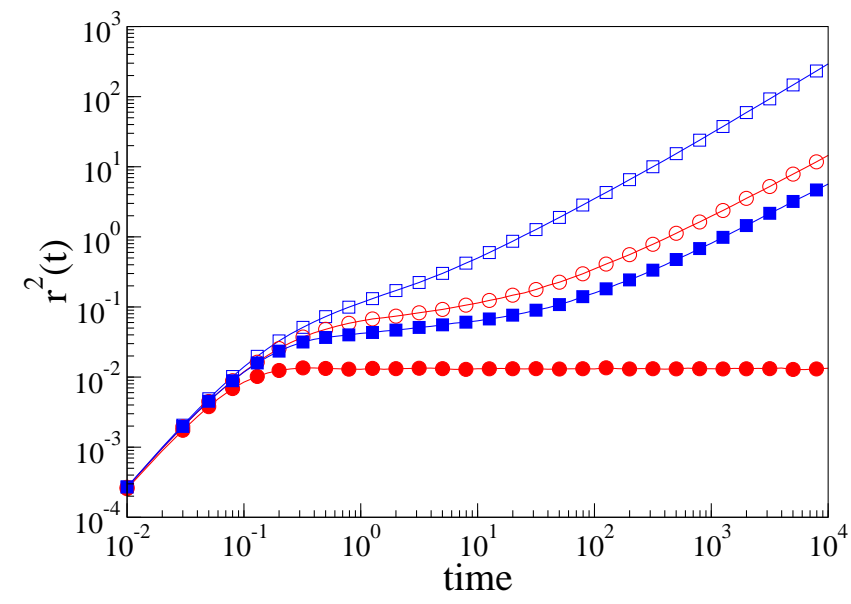

Fig. 5 The mean square displacement of the monomers in the gel (open symbols) is compared with that obtained when the spanning cluster is frozen (full symbols). Squares: $p=0.2, \phi=0.52$; Circles: $p=0.25, \phi=0.55$.

completely arrested by freezing the percolating cluster, showing that the relaxation of the system is completely due to the fluctuations of the percolating cluster. This result is relevant to model diffusion in biological systems, e.g. to investigate drug delivery processes.

\subsection{Correlation functions}

In this section, we describe the relaxation of the system in the gel phase through the study of the sISF, Eq. (2). Convention- 
ally, the relaxation time $\tau_{k}$ of the system is defined requiring that $F_{s}\left(k, \tau_{k}\right) \equiv 1 / e$, or a similar small value, and the transition of structural arrest is considered to occur when $\tau_{k}$ reaches a high value $\tau_{\text {arrest }}$, related the experimental time scale. Here, we fix $\tau_{\text {arrest }} \equiv 10^{5}$, and explicitly consider the dependence on the threshold used to define the relaxation time. Therefore, we derive a $k$ and $x$ dependent structural arrest line $\phi_{\text {arrest }}(p)$, defined as the volume fraction at which $F_{s}\left(k, \tau_{\text {arrest }}\right) \equiv x$.

Fig. 6(top panel) illustrates the dependence of $\phi_{\text {arrest }}$ on $x$ for three different values of $k$, at $p=0.3$. The figure clarifies the existence of two characteristic values for $\phi_{\text {arrest }}, \phi_{\text {gel }} \approx 0.4$ and $\phi_{\text {glass }} \approx 0.58$. Indeed, regardless of $x, \phi_{\text {arrest }} \approx \phi_{\text {gel }}$ at small $k$, and $\phi_{\text {arrest }} \approx \phi_{\text {glass }}$ at large $k$. At intermediate values of $k, \phi_{\text {arrest }}$ exhibits a crossover from $\phi_{\text {gel }}$ to $\phi_{\text {glass }}$ as $x$ increases. The decay of the correlation function at wavevectors $k_{\min }<k<k_{\max }$ is therefore influenced by both the gel and the glass transitions. The bottom panel of Fig. 6 illustrates the same crossover for different wavevectors, that influence the value of $x$ at which the crossover occurs. The glassy time scale affects the initial decay of the correlation function, and it is thus observed for large $x$, while the gel relaxation corresponds to the final relaxation, and it is observed for small $x$.

Data shown in Fig. 7 further clarify these findings. As also found in Ref. $\stackrel{10}{ }$, we can recognize three different relaxation time scales: $\tau_{\beta}$, due to the rattling of particles in the nearest neighbour cage, does not diverge at all; $\tau_{\alpha}$, due to the opening of the cage, diverging at the glass transition line; and finally $\tau_{\text {perc }}$, due to the relaxation of the largest cluster, diverging at the gel transition line (data plotted in Fig. 7 refer to $\phi>\phi_{g e l}$, then $F_{s}(k, t)$ does not relax to zero, and reaches at long time a finite value).

\section{Interference of structural arrest lines}

Here, we consider the interference of the glass and of the gel transitions in a model of a chemical gel, where bonds are permanent. As we have noticed, the glass transition is a discontinuous transition and the gel transition is a continuous one. Hence, we expect that the model system here studied might have some analogies with the mode coupling theory for schematic $F_{13}$ model $^{2}$, which gives two arrested lines: a continuous transition, which meets a discontinuous one. The model predict that the discontinuous transition ends on a high order critical point ( $A_{3}$ singularity), where the plateau of the two transitions coincide, characterized by logarithm decay $\underline{\underline{1}} \underline{\underline{3}}$ of the relaxation functions. Topologically the phase diagram is similar to the one found here (Fig. 1).

In our model, due to long relaxation time involved, it is rather difficult to localize such singularity. However we do find evidence of a logarithmic decay in a region inside the gel phase, close to the glass transition line, as shown in Fig. 8 .
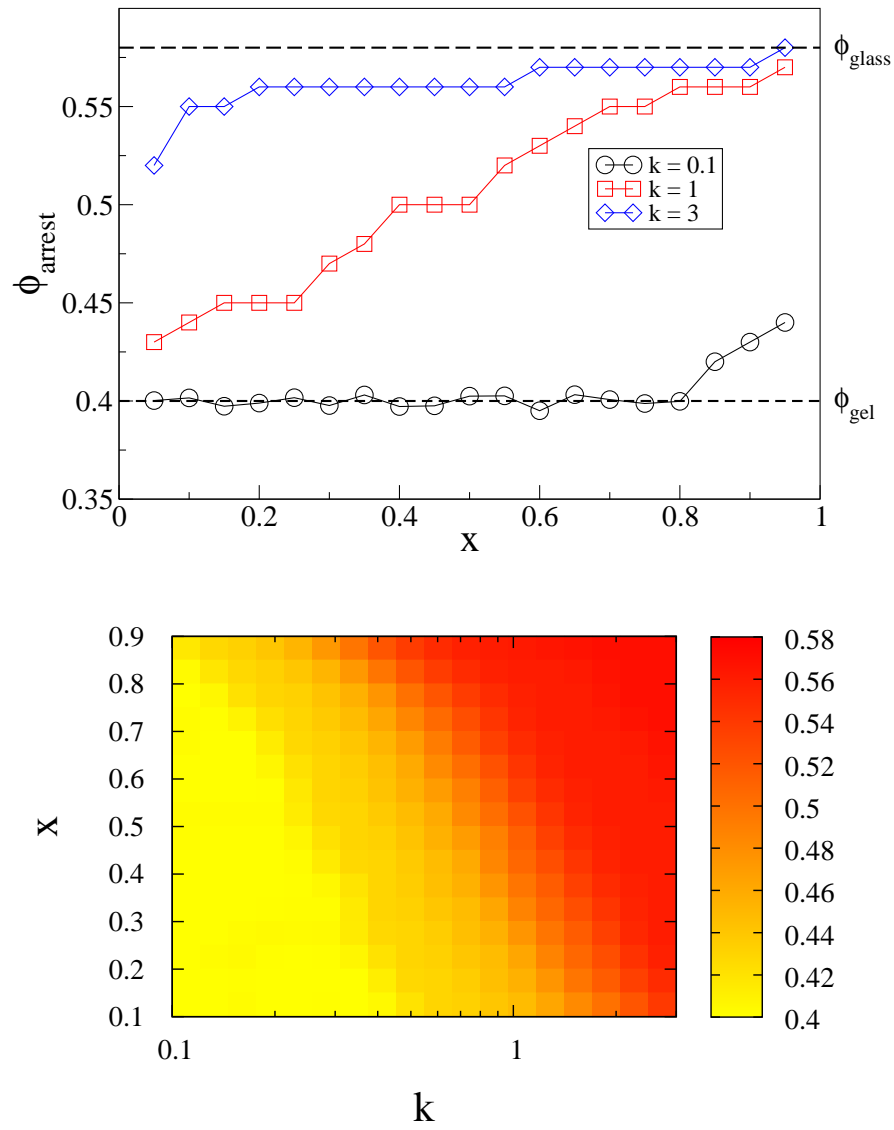

Fig. 6 We consider the transition of structural arrest to occur when $F_{S}\left(k, \tau_{\text {arrest }}\right) \equiv x$, with $\tau_{\text {arrest }} \equiv 10^{5}$. The top panel illustrates the dependence of $\phi_{\text {arrest }}$ on $x$, for three values of $k$, and $p=0.3$. The bottom panel is a colour map showing the dependence of $\phi_{\text {arrest }}$ on $k$, for $p=0.3$. The figures clarify that at small $k, \phi_{\text {arrest }} \approx \phi_{\text {gel }}$, at large $k, \phi_{\text {arrest }} \approx \phi_{\text {glass }}$, while at intermediate $k \phi_{\text {arrest }}$ exhibits a crossover between these two limiting values on increasing $x$.

In order to appreciate how this logarithmic decay emerges, we follow the evolution of the sISF on increasing $p$ along the glass line, in the gel phase. Fig. 9 shows that on increasing $p$ the value of the plateau associated to cage motion of the particles decreases, and the plateau becomes shorter. On increasing $p$ we also observe the value of the plateau associated to the gel transition to grow, and the time needed to reach it to increase. Accordingly, the higher order critical point where a logarithm decay is found seems to be associated to the state where the two plateau coincide, in agreement with the $A_{3}$ singularity of the $F_{13}$ model. We stress that the high order singularity may occur at higher values of $p$ including possibly $p=1$. Further investigation is necessary. 


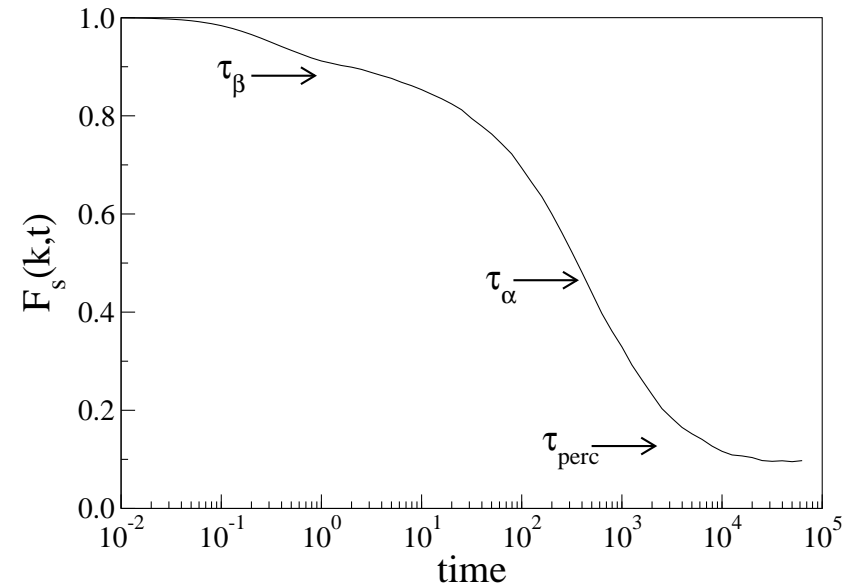

Fig. 7 The $\operatorname{siSF}, F_{S}(k, t)$, for $k=3, p=0.3$, and $\phi=0.54$, larger than $\phi_{\text {gel }} \approx 0.4$. The three relaxation processes are clearly distinguishable.

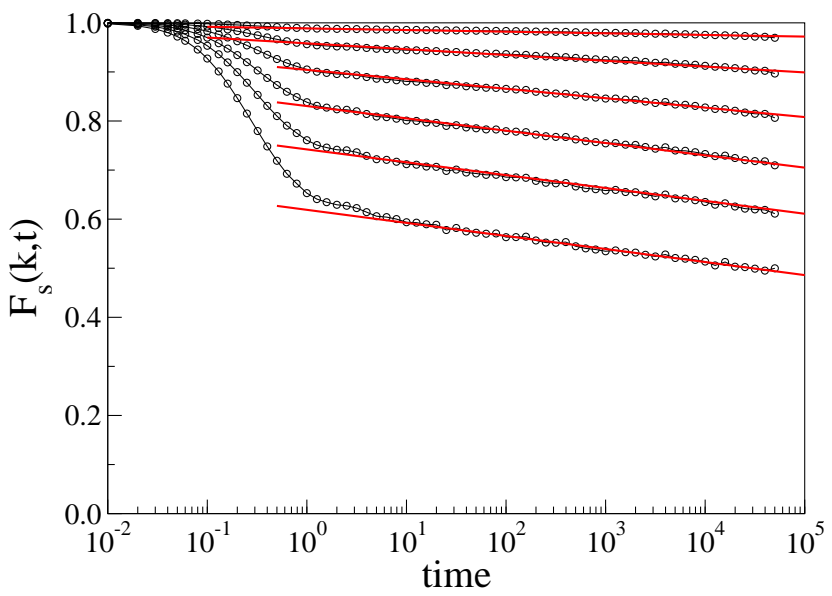

Fig. 8 The $\operatorname{siSF}, F_{S}(k, t)$, for $p=0.5, \phi=0.52$, and wavevector $k=1,2,3,4,5,6.28$ (from top to bottom). Continuous lines are logarithmic functions.

\section{Conclusions}

The gel and the glass transitions are characterized by different dynamical signatures, reflecting the fact that the gel transition is a continuous transition marking the arrest of the dynamics on the largest length scale of the system, while the glass transition is a discontinuous transition marking the arrest of the dynamics at all length scales. This makes of interest the investigation of the interplay of these transitions in polymeric suspensions, where the transition are driven by an increase of the cross-linker concentration and the polymer volume fraction. Here, we have performed such an investigation, focusing on the glassy dynamics within the gel phase and on their mutual interference.

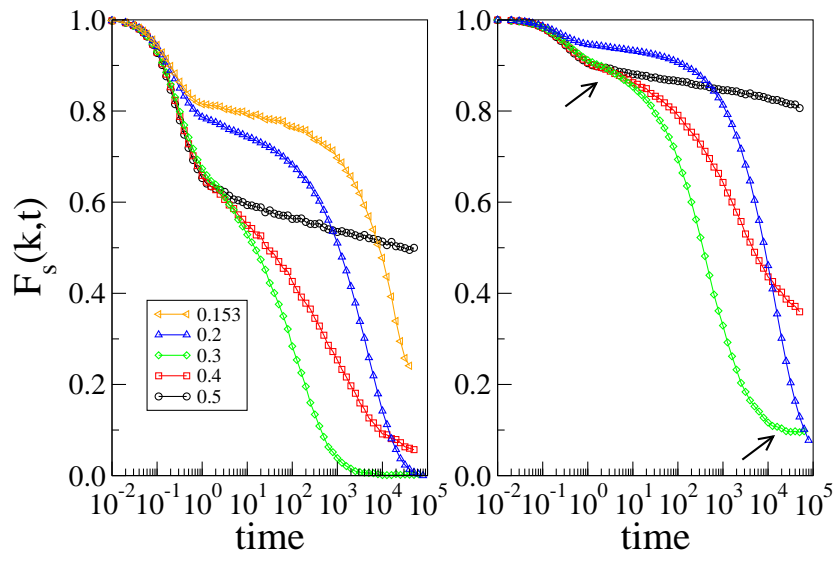

Fig. 9 The sISF, $F_{S}(k, t)$, at different values of $p$ as indicated, moving along the glass line, for $k=k_{\max }$ (left panel) and for $k=3$ (right panel). The plateau associated with the gel and the glass are indicated by an arrow.

An important open question ahead regards the relevance of the mode coupling theory for schematic $F_{13}$ model $^{2}$ for these systems. Our results support this scenario, as we do not only recover the interference of a continuous and of a discontinuous transition, but we also find a point where the relaxation function seems to decay logarithmically, which should be associated to the A3 singularity. However, more work is needed in this direction, particularly to better localize this high order critical singularity.

\section{Acknowledgement}

The authors would like to thank Dr. M. Sellitto for having drawn their attention on the properties of the $F_{13}$ model.

\section{References}

1 K. Dawson, G. Foffi. M. Fuchs, W. Gotze, F. Sciortino, M. Sperl, P. Tartaglia, T. Voigtmann, and E. Zaccarelli, Phys. Rev. E 63, 011401 (2000)

2 W. Götze and and M. Sperl, Phys. Rev. E 66, 011405 (2002)

3 W. Götze, Complex dynamics of glass-forming liquids, Oxford University Press, Oxford, 2009

4 T. Eckert and E. Bartsch, Phys. Rev. Lett. 89, 125701 (2002)

5 K. N. Pham, A. M. Puertas, J. Bergenholtz, S. U. Egelhaaf, A. Moussaid, P. N. Pusey, A. B. Schofield, M. E. Cates, M. Fuchs, W. C. K. Poon, Science 296, 104 (2002)

6 S. H. Chen, W. R. Chen, F. Mallamace, Science 300, 619-622 (2003)

7 A. M. Puertas, M. Fuchs, and M. E. Cates, Phys. Rev. Lett. 88, 098301 (2002)

8 F. Sciortino, P. Tartaglia, E. Zaccarelli. Phys. Rev. Lett. 91268301 (2003)

9 X. H. Lu, S. G. J. Mochrie, S. Narayanan, A. R. Sandy, and M. Sprung Phys. Rev. Lett. 100, 045701 (2008)

10 P. I. Hurtado, L. Berthier, and W. Kob, Phys. Rev. Lett. 98135503 (2007); P. Chaudhuri, L. Berthier, P. I. Hurtado, and W. Kob, Phys. Rev. E 81, 040502(R) (2010) 
11 M.P. Allen and D.J. Tildesley, Computer Simulation of Liquids, Oxford University Press (1987)

12 B. D. Lubachevsky, Journal of Computational Physics Volume 94, 255 (1991)

13 Ning Xu, Thomas K. Haxton, Andrea J. Liu, and Sidney R. Nagel, Phys. Rev. Lett. 103, 245701 (2009)

14 L. Berthier and T. A. Witten, Phys. Rev. E 80, 021502 (2009)

15 P. Chaudhuri, L. Berthier, and S. Sastry, Srikanth, Phys. Rev. Lett. 104, $165701(2010)$

16 C.S. O'Hern, S. A. Langer, A. L. Liu, and S. R. Nagel, Phys. Rev. Lett. 88, 075507 (2002)

17 P. J. Flory, The Physics of Polymer Chemistry (Cornell University Press, Ithaca, NY, 1954)

18 P. G. de Gennes, Scaling Concepts in Polymer Physics (Cornell University Press, Ithaca, NY, 1993)

19 D. Stauffer and A. Aharony, Introduction to percolation theory (Taylor $\&$ Francis, London, 1992).

20 J. E. Martin and J. P. Wilcoxon, Phys. Rev. Lett. 61, 373 (1988); J. E. Martin, J. Wilcoxon, and J. Odinek, Phys. Rev. A 43, 858 (1991)

21 P. Lang and W. Burchard, Macromolecules 24, 814 (1991)

22 S. Z. Ren and C. M. Sorensen, Phys. Rev. Lett. 70, 1727 (1993)

23 E. Del Gado, A. Fierro, L. de Arcangelis, and A. Coniglio, Phys. Rev. E 69, 051103 (2004)

24 T. Abete, A. de Candia, E. Del Gado, A. Fierro, A. Coniglio, Phys. Rev. Lett. 98088301 (2007)

25 T. Abete, A. de Candia, E. del Gado, A. Fierro, and A. Coniglio, Phys. Rev. E 78, 041404 (2008)

26 A. Fierro and T. Abete and A. Coniglio, J. Chem. Phys. 13119 (2009)

27 M. Goldbart, H. E. Castillo, and A. Zippelius, Adv. Phys. 45, 393 (1996); C. Wald, P. M. Goldbart, and A. Zippelius, J. Chem. Phys. 124, 214905 (2006)

28 A. Fierro, T. Abete, A. de Candia, E. Del Gado, and A. Coniglio, J. Phys.: Condens. Matter 21, 504110 (2009)

29 H. A. Lorentz, Proc. Amst. Acad. 7, 438 (1905).

30 J. Kurzidim, D. Coslovich, and G. Kahl, J. Phys.: Consens. Matter 23, $234122(2011)$

31 S. Babu, J. C. Gimel, and T. Nicolai, J. Phys. Chem. B 112, 743 (2008)

This journal is ๑ The Royal Society of Chemistry [year]

Journal Name, 2010, [vol], $1-7$ | 7 